\title{
Effect of Exergaming on Muscle Strength, Pain, and Functionality of Shoulders in Cancer Patients
}

\author{
Paulo Furtado de Oliveira, MSc, ${ }^{1}$ Ricardo da Silva Alves, MSc, ${ }^{2}$ Denise Hollanda lunes, PhD, ${ }^{1}$ \\ Jovana Maria de Carvalho, PT, Juliana Bassalobre Carvalho Borges, PhD, \\ Flávia da Silva Menezes, $\mathrm{PT},{ }^{1}$ and Leonardo César Carvalho, $\mathrm{PhD}^{1,2}$
}

\begin{abstract}
Objective: To compare the strength of the middle deltoid muscle by means of dynamometry and the Shoulder Pain and Disability Index (SPADI) in participants in treatment for cancer after the practice of an exergaming protocol.

Materials and Methods: We conducted a randomized controlled clinical trial. Thirty-eight voluntary participants were divided into two groups: cancer group $(n=19$; age $=61.46 \pm 8.79$ years; body mass index $[\mathrm{BMI}]=$ $\left.28.36 \pm 4.94 \mathrm{~kg} / \mathrm{m}^{3}\right)$ and control group $\left(n=19\right.$; age $=57.62 \pm 7.57$ years; BMI $\left.=28.06 \pm 3.74 \mathrm{~kg} / \mathrm{m}^{3}\right)$, and they participated in the study. All participants performed an exergame protocol by using Xbox $360 \mathrm{Kinect}^{\circledR}$ (Microsoft, Redmond) with the game Your Shape Fitness Evolved 2012 two to three times per week for 20 sessions. They were evaluated through the isometric dynamometer in the middle deltoid muscle and the SPADI at three moments: preintervention (EV1), after 10 sessions (EV2), and after 20 sessions (EV3).

Results: The cancer group scored higher on both the disability index, in all three evaluations, and the pain index, in EV2 and EV3, when compared with the control group. There was a significant reduction in the disability score in EV2 and EV3 when compared with EV1 in the cancer group, whereas pain was lower in EV2 and EV3 when compared with EV1 in the control group. There were no significant interevaluation or intergroup differences in the maximal isometric voluntary contraction of the deltoid muscle of both upper limbs.

Conclusion: At the end of the exergaming, protocol reduced the disability of the shoulder joint of the cancer group and decreased the differences between the groups for disability and pain scores, without changing isometric force.
\end{abstract}

Keywords: Shoulders, Exergaming, Cancer, Rehabilitation

\section{Introduction}

C ANCER IS THE third leading cause of death in developing countries. ${ }^{1}$ Fatigue is the most prevalent side effect of cancer treatment. $^{2,3}$ This symptom prevails from the beginning to the end of the treatment, persisting for years and negatively influencing quality of life. ${ }^{3-5}$ Cancer-related fatigue (CRF), according to the National Comprehensive Cancer Network, "is a distressing, persistent and subjective sense of physical, emotional, and/or cognitive tiredness or exhaustion related to cancer or cancer treatment that is not proportional the recent activity performed and interferes with usual functioning." 6 This condition affects cognitive abilities, characterized by reduced motivational levels, interest, attention, and concentration, as well as memory loss, ${ }^{5}$ leading to the impairment of physical performance. ${ }^{2}$
CRF is divided into central fatigue and peripheral fatigue. Central fatigue is characterized by failure of the central nervous system to transmit neuromotor impulses, such that the muscle does not reach its maximum capacity of voluntary contraction. ${ }^{7,8}$ Peripheral fatigue occurs under conditions of excessive physical exertion, compromising the muscle excitation-contraction mechanism ${ }^{8}$ and altering the propagation of the action potential and excitability of the muscular membrane. ${ }^{7}$ These factors result in a low voluntary capacity to produce strength to power the muscle, ${ }^{7,8}$ reducing the muscle's ability to perform motor tasks.

Associated with treatments such as chemotherapy and radiotherapy, fatigue symptoms have a prevalence of up to $96 \%$ among patients being treated for cancer. ${ }^{3}$ These treatment modalities may include other short- and long-term complications, such as a reduction of the shoulder function,

\footnotetext{
${ }^{1}$ Motricity of Sciences Institute, and ${ }^{2}$ Bioscience Program, Federal University of Alfenas, Alfenas, Brazil.
} 
an increase in pain, a decrease in range of motion, numbness, and strength of the muscle. ${ }^{9}$ The surgical procedures may cause an accessory spinal nerve injury, with a consequent shoulder dysfunction, especially in the upper limb abduction movement. ${ }^{10}$ In cancer patients, sensory and motor impairments were observed, which affect the nerve impulse conduction velocity, ${ }^{11}$ resulting in loss of muscle strength. ${ }^{12}$ Thus, these conditions may compromise the shoulder ability to perform daily life activities, especially caused by the decline of the middle deltoid muscle force, which has increased its activation in the first $90^{\circ}$ of shoulder abduction. ${ }^{13}$ Thus, the shoulder joint morbidity can remain for up to 6 years after the surgery, compromising the daily living activities, including the ability to return to work. ${ }^{14,15}$ Considering its prevalence in people with cancer, many health professionals seek to understand and develop safe and effective techniques to reduce these symptoms. ${ }^{15}$

The main treatment techniques for CRF complaints are psychotherapy, physiotherapy, nutritional adequacy, therapy for sleep disturbances, and pharmacological treatments. ${ }^{16}$ Physical exercise can reduce the intensity of this symptom, with relief being proportional to the volume of weekly practice. ${ }^{17,18}$ Physical exercise programs improve physical fitness, quality of life, aerobic capacity, muscular strength, depression, and well-being ${ }^{18-20}$ The American College of Sports Medicine recommends that, to apply exercise safely to cancer patients, exercise intensity should start low and increase according to the individual's ability. ${ }^{20}$

Exergaming, which is exercising through videogames, requires attention and motivation, promotes a sense of accomplishment, and, being pleasurable, contributes to adherence to treatment. ${ }^{21}$ Exergaming can reproduce the practice of light- to moderate-intensity physical activity when used with combined whole-body movements. ${ }^{22}$ Therefore, exergaming is a viable alternative for the treatment of CRF. ${ }^{17}$ The aim of this study was to determine the effects of exergaming on the production of strength in the middle deltoid muscle and on functionality and pain in shoulder joints of cancer patients.

\section{Materials and Methods}

\section{Study design}

This is a controlled, quasi-experimental study conducted between January 2015 and October 2016. The study was approved by the Research Ethics Committee of the Federal University of Alfenas (Protocol no. or CAAE no. 51644515.7.0000.5142) and registered in the Registration Platform of Clinical Trials (RBR9t48g5). All volunteers were informed of the procedures involved in the study; on their agreement to participate, they signed the informed consent form.

\section{Participants}

The sample used in this study is part of the larger study, dealing with effects of exergaming in CRF in the quality of life and electromyography of the middle deltoid of people with cancer in treatment: a controlled trial. ${ }^{23}$ In that study, quality of life data and middle deltoid muscle electromyography of cancer patients were analyzed. In this study, the maximal voluntary isometric contraction (MVIC) of the middle deltoid muscle and disability and pain data, as measured through the Shoulder Pain and Disability Index (SPADI), are presented.

The inclusion criteria for the cancer group were: patients between 18 and 80 years old with a good ability to understand the evaluation and training methods, clinical diagnosis of any type of cancer in stage 0 to III, who had or had not undergone radiotherapy or chemotherapy, and who obtained medical release for the practice of physical activity. The inclusion criteria were the same for the control group, except for the cancer diagnosis and treatment.

The exclusion criteria were as follows: presence of orthopedic lesion in the shoulder with signs and symptoms active in the past 6 months, presence of nerve damage that impairs upper limb motility, reduced limb activity that prevents participation in the exergaming protocol, presence of infectious disease, presence of cutaneous lesions that make evaluation impossible, incapable of voluntary movement of the upper limbs, and incapable of performing isotonic exercises. Those who, for any reason, refused to sign the free and informed consent form were also excluded.

\section{Assessment procedures}

All evaluations and trainings were carried out by the same evaluator at the Human Movement Analysis Laboratory of the Federal University of Alfenas-Minas Gerais (LAMUnifal-MG). A preliminary evaluation was carried out via a sociodemographic and clinical questionnaire. Then, an inspection and palpation were performed on the upper limb, anterior trunk, and posterior trunk to observe any changes, such as hyperemia, cyanosis, spots, fibrosis, feverish state, or any other dermal alteration.

MVIC was obtained by an EMG System ${ }^{\circledR}$ model 830c (EMG System, São José dos Campos, São Paulo, Brazil), which was equipped with a load cell with a total capacity of $200 \mathrm{kgf}$. For the analysis, three maximal isometric contractions were performed, with a duration of 60 seconds for each contraction and a rest period of 5 minutes between each task. ${ }^{23}$ Before the test, the evaluator issued a statement to all the volunteers to instruct them both verbally and visually regarding the proper way to perform the test.

Each volunteer was placed in a seated position in a rigid chair, with the back supported on the backrest, with the upper limb to be tested in an abduction of $\sim 90^{\circ}$, aligned with the trunk, a position in which the middle deltoid muscle has great activation. ${ }^{13}$ All were given the same incentive to perform abduction of the shoulder against the load cell, sustaining maximum force throughout the test for 60 seconds. The volunteer was verbally encouraged to achieve maximum strength with the phrase, "Strength, strength, strength...." The test was ended if the volunteer could not keep MVIC for 60 seconds after the beginning of the test. ${ }^{24}$

For the MVIC analysis, the first five and the last five seconds were excluded to minimize the effect of the verbal encouragement until isometric contraction was reached, obtaining a 50-second test interval. The mean of the three trials was considered an estimate of the MVIC. ${ }^{25}$ For this analysis, researchers used the EMGLab v.1 software (EMG System do Brazil).

To evaluate participants' pain level and functionality, the SPADI questionnaire, translated and adapted into the 
Portuguese language, ${ }^{26}$ was used. The questionnaire consists of 13 questions, 8 on disability and 5 on pain, in which the patients are asked to rate the difficulty level, from 0 to 10 , they faced when performing 10 activities during the previous week. After completing the questionnaire, the score for each domain (disability and pain) is given in a percentage, such that the higher its value, the greater the pain and dysfunction. ${ }^{26}$

After the first evaluation, participants completed ten exergaming sessions, followed by a second evaluation. Then came another ten sessions of the proposed exergaming protocol, and, finally, a third evaluation using the same evaluation instruments.

\section{Exergaming protocol}

The exergaming protocol was performed in the Human Movement Analysis Laboratory of the Federal University of Alfenas (LAM), which is equipped with eight 42-inch LED TVs and XBox360 Kinect consoles (Microsoft). The study used the game Your Shape Fitness Evolved 2012 (Ubisoft, Canada). The two groups participated in exergaming sessions.
The practice of exergaming not only sought to follow the recommendations proposed by the American College of Sports Medicine for physical exercise prescription in cancer patients, who require 150 minutes of moderate-intensity exercise, 75 minutes of vigorous exercise, or an equivalent combination of both, per week, but also sought to meet the individual needs of each patient. $^{20}$

The chosen games within Your Shape Fitness Evolved 2012 were Wall Breaker, Stomp It, and Run the World. The game Wall Breaker has the objectives of improved coordination, agility, and velocity of breaking blocks with the upper limbs. Players are scored as a function of execution time: The faster the execution, the higher the score. The objective of Stomp It is to step on the lights that appear around the patient by performing anterior and lateral movements of the lower limbs, adjusting the position of the arms during the movements of the lower limbs, to remain in orthostatic postural balance. This game has the objective to improved coordination, agility. The game Run the World, which simulates distance walking of $400 \mathrm{~m}$, had its condition determined by the game, performing knee and hip flexion movements. The objective of this game is to promote

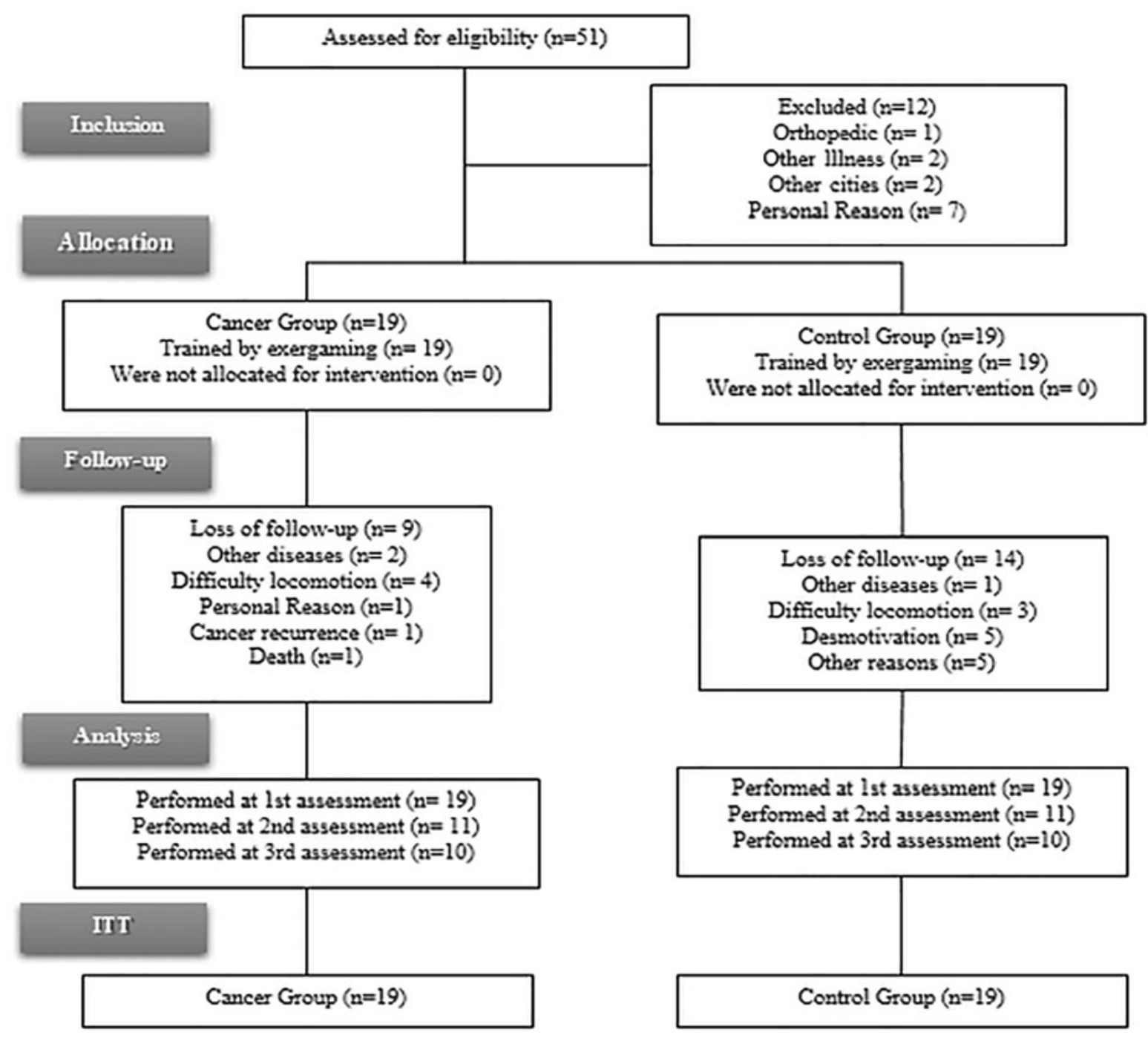

FIG. 1. Study design. CONSORT diagram. 
improved cardiovascular fitness and well-being. This game was added to increase workload and minimize any possible disinterest of the participants.

\section{Statistical analysis}

The Statistical Package for Social Sciences (SPSS) (IBM Corp., Chicago), version 20.0 was used for statistical analysis of the data. The variables were analyzed by means of the intention-to-treat method, illustrated in the flow chart of the study design shown in Figure 1. Initially, the data were analyzed by using descriptive statistical methods, obtaining the values of the mean, standard deviation, and confidence intervals. Then, all datasets were tested for normality by using the Kolmogorov-Smirnov test, with significance set at $P<0.05$. To analyze differences in the variables age and body mass index (BMI), a one-way analysis of variance (ANOVA) was used.

The effect of the intervention was compared between the groups by means of an ANOVA two-way repeated measures model. The ANOVA was submitted to Mauchly's sphericity test. In cases of sphericity violation Huynh-Feldt correction was adopted. The post hoc Bonferroni test was used to compare inter- and intragroup analysis. A significance level of $5 \%$ was set for all variables.

The effect size was calculated by using $\mathrm{G}^{*}$ Power software, v. 3.1.7 (Franz Faul, Universität Kiel, Germany). To calculate the effect size in ANOVA, $\mathrm{f}^{2}$ Cohen was used and values from 0.02 to 0.15 (small effect), 0.15 to 0.35 (median effect), and above 0.35 (large effect) were adopted. For the sample power, values above 0.80 were considered as having high power, and a significance level of $\alpha=0.05$ was applied.

\section{Results}

The sociodemographic and clinical characteristics of the sample of this study are presented in Table 1 . The one-way ANOVA revealed no significant differences between the groups with respect to age and BMI. A chi-square test revealed no significant differences with respect to sex $(P=0.426)$ and physical activity $(P=0.721)$

The shoulder disability and pain scores are shown in Table 2. The cancer group obtained higher score values than the control did during all evaluations for the disability index and in the two last evaluations for the pain index. Therefore, the highest SPADI scores in the cancer group may be linked to the current cancer treatment.

With the exergaming intervention in the cancer group, there was a significant reduction in disability observed in the SPADI questionnaire scores between the assessments. The exergaming protocol did not reduce the level of pain by the SPADI questionnaire in the cancer group, whereas there was a significant reduction in the level of pain in the control group.

Table 3 shows the means and standard deviations of the maximal voluntary isometric contraction (MVIC) of the medial deltoid muscle on both sides at each evaluation. There were no significant differences between the evaluations or between the two groups.

\section{Discussion}

The SPADI questionnaire, which has been validated for the Portuguese language ${ }^{26}$ is widely used to assess function
Table 1. Socio-Demographic Characteristics of Study Participants

\begin{tabular}{|c|c|c|c|}
\hline Characteristics & $\begin{array}{c}\text { Cancer } \\
\text { group } \\
(\mathrm{n}=19)\end{array}$ & $\begin{array}{c}\text { Control } \\
\text { group } \\
(\mathrm{n}=19)\end{array}$ & $\mathrm{P}$ \\
\hline Age (years) & $61.46 \pm 8.79$ & $57.62 \pm 7.57$ & 0.301 \\
\hline Body mass index & $28.36 \pm 4.94$ & $28.06 \pm 3.74$ & 0.844 \\
\hline \multicolumn{4}{|l|}{ Sex } \\
\hline Male (\%) & $15.79(n=3)$ & $26.32(n=5)$ & 0.426 \\
\hline Female $(\%)$ & $84.21(n=16)$ & $73.68(n=14)$ & \\
\hline $\begin{array}{l}\text { Cancer diagnosis } \\
\text { (months) }\end{array}$ & $49.78 \pm 39.40$ & & \\
\hline $\begin{array}{l}\text { Performs physical } \\
\text { activity }(\%)\end{array}$ & $26.32(n=5)$ & $31.58(n=6)$ & 0.721 \\
\hline Smoking $(\%)$ & 0 & $10.52(n=2)$ & \\
\hline Alcoholism (\%) & 0 & $5.26(n=1)$ & \\
\hline \multicolumn{4}{|c|}{ Cancer diagnosis site $(\%)$} \\
\hline $\begin{array}{l}\text { Gastrointestinal } \\
\text { tract }\end{array}$ & $10.52(n=2)$ & & \\
\hline Breast & $47.37(n=9)$ & & \\
\hline Abdomino-pelvic & $5.26(n=1)$ & & \\
\hline Ovary & $10.52(n=2)$ & & \\
\hline Uterus & $10.52(n=2)$ & & \\
\hline Prostate & $10.52(n=2)$ & & \\
\hline Oropharynx & $10.52(n=2)$ & & \\
\hline \multicolumn{4}{|l|}{ Stage $(\%)$} \\
\hline 0 & 0 & & \\
\hline I & $15.79(n=3)$ & & \\
\hline II & $47.37(n=9)$ & & \\
\hline III & $10.52(n=2)$ & & \\
\hline IV & 0 & & \\
\hline No data & $26.32(n=5)$ & & \\
\hline \multicolumn{4}{|c|}{ Chemotherapeutic drug $(\%)$} \\
\hline Antibiotics & $11.1(n=1)$ & & \\
\hline Platinum & $22.2(n=2)$ & & \\
\hline Taxanes & $66.7(n=6)$ & & \\
\hline \multicolumn{4}{|c|}{ Types of treatment performed $(\%)$} \\
\hline Just surgery & $5.26(n=1)$ & & \\
\hline Just chemotherapy & 0 & & \\
\hline Just radiotherapy & $10.52(n=2)$ & & \\
\hline $\begin{array}{l}\text { Surgery and } \\
\text { chemotherapy }\end{array}$ & $21.05(n=4)$ & & \\
\hline $\begin{array}{l}\text { Surgery and } \\
\text { radiotherapy }\end{array}$ & $5.26(n=1)$ & & \\
\hline $\begin{array}{l}\text { Chemotherapy } \\
\text { and radiotherapy }\end{array}$ & $5.26(n=1)$ & & \\
\hline All & $52.65(n=10)$ & & \\
\hline
\end{tabular}

Mean \pm standard deviation; $\%$, percentages; $n$, number of members.

and pain in the shoulder joint in patients with various conditions, such as acute pain in the shoulder joint, ${ }^{27}$ resection of head and neck cancer, ${ }^{10,28}$ mastectomies, ${ }^{9,29}$ and frozen shoulder. $^{30}$

The volunteers in the cancer group had no clinical diagnosis of injury to the shoulder joint, but they presented higher SPADI scores than the control group, which suggests greater disability and pain for the former. Scores obtained by the cancer group resemble clinical shoulder lesions such as subacromial impact syndrome, rotator cuff tendinopathies, and frozen shoulder syndrome. ${ }^{30-32}$ In this study, there was an incidence of $47.37 \%$ breast cancer, and $84.20 \%$ of these 
Table 2. Comparative Analysis of Scores (Mean \pm Standard Deviation) Obtained in the Shoulder Pain and Disability Index Questionnaire of Cancer and Control Groups at the Moment of Evaluation

\begin{tabular}{llccccccc}
\hline & & & & & \multicolumn{3}{c}{ ANOVA-P } \\
\cline { 6 - 8 } Variable & Groups & EV1 & EV2 & EV3 & $G^{*} E V$ & Evaluation & Group & ES \\
\hline SPADI dis & Cancer & $28.25 \pm 21.80^{\mathrm{a}}$ & $17.22 \pm 18.76^{\mathrm{ab}}$ & $18.48 \pm 20.91^{\mathrm{ab}}$ & 0.003 & 0.009 & 0.001 & 0.628 \\
& Control & $3.68 \pm 5.45$ & $4.34 \pm 7.01$ & $4.34 \pm 7.01$ & $F=7.670$ & $F=5.969$ & $F=14.175$ & \\
SPADI pain & Cancer & $33.78 \pm 33.87$ & $28.10 \pm 27.78^{\mathrm{a}}$ & $28.84 \pm 32.34^{\mathrm{a}}$ & 0.853 & 0.176 & 0.002 & 0.571 \\
& Control & $8.52 \pm 12.12$ & $5.36 \pm 8.32^{\mathrm{b}}$ & $5.36 \pm 8.32^{\mathrm{b}}$ & $F=0.127$ & $F=1.816$ & $F=11.775$ & \\
\hline
\end{tabular}

${ }^{a}$ In the same column represents statistical difference intergroups.

${ }^{\mathrm{b}}$ In the same row represents statistical difference intragroups by Bonferroni test.

ANOVA, analysis of variance; ES, effect size; EV, evaluation; G*EV, interaction groups and evaluations; SPADI, Shoulder Pain and Disability Index; SPADI dis., SPADI disability.

patients had undergone some surgery; similar scores have been found in post-mastectomy patients in the immediate postoperative period. ${ }^{29}$ Therefore, the results suggest that even without any type of diagnosed lesion, there may be functional impairment of the shoulder joint.

Mastectomy has physical and functional repercussions for this population, such as loss of strength, decreased limb movement capacity, and decreased function. ${ }^{29,33}$ In addition, higher levels of pain are associated with the surgical procedure and with treatments such as chemotherapy and radiotherapy ${ }^{34}$ Therefore, the highest SPADI scores in the cancer group may be linked to current treatment strategies. Thus, this study reinforces the need to pay more attention to the general conditions of this population.

Another possible cause of shoulder dysfunction may be surgical procedures given as treatment for neck and head cancers, as these may lead to injury to the accessory spinal nerve, generating dysfunction in this region, including with the movement of abduction. ${ }^{10}$ These cancer patients had the highest SPADI scores. ${ }^{28}$

Another hypothesis for why SPADI scores are higher in this population is an apparent lack of motivation and increased perception of effort, which could be associated with psychosocial factors, such as avoidance and catastrophizing pain. ${ }^{35}$ This suggests that in volunteers with cancer, volunteers' belief that they are less able to perform actions, such as raising their arm to perform activities or carrying heavy objects, could interfere with their performance during the questionnaire-based evaluation, even when no MIVC changes are found through isometric dynamometer. This condition has been described in the literature: The highest SPADI scores for pain and disability were observed in patients with chronic shoulder pain, and the fact that isokinetic strength had not changed was not related to the scores obtained in the questionnaire. ${ }^{36}$

The exergaming protocol used was able to reduce the disability subscale scores in the cancer group, although they have not yet reached levels similar to those of the control group. Other studies have also demonstrated a reduction in SPADI scores after the conventional exercises. ${ }^{10,29}$ Also, exergaming proved to be an effective treatment for some types of shoulder joint dysfunction, such as impact syndrome and joint pain, when compared with conventional treatment. ${ }^{37}$ Shoulder pain in healthy individuals is common, occurring in at least one in three people at some point in their life, ${ }^{38}$ and underdiagnosis of lesions may also occur. The protocol proposed in this study may not have been enough to significantly reduce the levels of referred pain in shoulders of the cancer group. A possible explanation for this apparent absence of modification in pain perception in the cancer group may be chemotherapy-induced neuropathy and central sensitization mechanisms, ${ }^{39}$ although we have not evaluated these.

Peripheral neuropathy induced by chemotherapy occurs in $\sim 68 \%$ of patients undergoing chemotherapy after the end of treatment and may be present in almost $30 \%$ of individuals 6 months after completion of treatment, compromising their quality of life and functional activities. ${ }^{39}$ Another possible explanation is central sensitization, manifested by increased responsiveness of nociceptive neurons in the central nervous system. In this case, natural, healthy movement that is not threatening can be perceived as threatening, enhancing the individual's pain experience. ${ }^{39}$ The absence of modified pain levels in the cancer group may also reflect psychosocial influences (such as avoidance of fear, catastrophic pain), which may cause the development

Table 3. Comparative Analysis of Maximal Isometric Voluntary Contraction Values (KgF) (Mean \pm Standard Deviation) of the Middle Deltoid Muscles in the Cancer and Control Groups at the Moment of Evaluation

\begin{tabular}{lcccccccc}
\hline Member & Groups & EV1 & EV2 & EV3 & $G^{*} E V$ & Evaluation & Group & ES \\
\hline $\mathrm{R}$ & Cancer & $2.74 \pm 1.07$ & $2.88 \pm 1.03$ & $2.59 \pm 0.99$ & 0.632 & 0.256 & 0.107 & 0.276 \\
& Control & $3.27 \pm 1.09$ & $3.25 \pm 0.89$ & $3.16 \pm 0.88$ & $F=0.462$ & $F=1.387$ & $F=2.734$ & \\
$\mathrm{~L}$ & Cancer & $2.58 \pm 1.06$ & $2.50 \pm 1.06$ & $2.60 \pm 1.07$ & 0.770 & 0.834 & 0.350 & 0.156 \\
& Control & $2.91 \pm 1.27$ & $2.88 \pm 1.15$ & $2.85 \pm 1.11$ & $F=0.174$ & $F=0.108$ & $F=0.350$ & \\
\hline
\end{tabular}

L, left; R, right. 
and maintenance of pain sensitization. ${ }^{36}$ Therefore, it is necessary to be aware of these factors to approach the treatment of shoulder dysfunction more successfully.

Commonly, patients undergoing chemotherapy and/or radiotherapy show reduced muscle strength when compared with healthy patients. ${ }^{14}$ Individuals who undergo treatment for breast cancer show loss of strength in the muscles in the shoulder joint, ${ }^{34}$ as well as negative effects from the treatment of cancer in the head or neck. ${ }^{10}$ However, a study analyzing cancer patients with or without CRF found that muscle strength in the biceps brachii did not change in cases where fatigue was present, ${ }^{40}$ corroborating our findings for the deltoid muscle. In our study, both groups demonstrated similar values for isometric strength, though the heterogeneity of the types of cancer may have influenced the results.

Muscle strength production correlates with sex, being higher in males. With advancing age, both groups did not show differences in MVIC. Patients with cancer previously treated with chemotherapy and then with radiotherapy, when participating in a progressive resistance training program, increased their capacity in the muscles of the upper limbs to generate force. ${ }^{41}$ Due to the lack of resistance during exercise, exergaming does not compensate for the loss of strength that occurs during the period of chemotherapy and/or radiotherapy.

This study has some limitations. First, we have not controlled for the possible etiology of the fatigue symptom, such as changes in hematological factors. Second, because this study did not take place in a cancer referral center, it was very difficult to obtain a satisfactory sample, which could have influenced the results. This explains the heterogeneity of the cancers obtained in the cancer group, as well as the types of treatment performed on these volunteers. In addition, an analysis of the intensity of physical activity during the exergaming practice could have been used to monitor volunteers' responses to the effort. Future studies will be needed to better elucidate these still obscure issues.

\section{Conclusion}

The differences initially found in shoulder joint dysfunction in the cancer group were reduced after the exergaming intervention protocol. However, after the intervention, there were no significant changes in the perceived pain level in the shoulder joint of the cancer group. The production of isometric muscle strength of the middle deltoid muscle was similar between groups, remaining unchanged after the performance of the proposed protocol.

\section{Acknowledgments}

The authors express their gratitude to Santa Casa de Alfenas and Federal University of Alfenas.

\section{Author Disclosure Statement}

No competing financial interests exist.

\section{Funding Information}

The authors are grateful to Santa Casa de Alfenas and the Foundation for Research of the State of Minas GeraisFAPEMIG, under APQ: 03580-13, and the Oncology Network of the State of Minas Gerais-FAPEMIG, under RED-11-14, for their financial support.

\section{References}

1. World Health Organization. Global action against cancer. 2006. www.who.int/cancer/media/en/GlobalActionCancer Englfull.pdf (accessed September 28, 2018).

2. Jones JM, Olson K, Catton P, et al. Cancer-related fatigue and associated disability in post-treatment cancer survivors. J Cancer Surviv Res Pract 2015; 10:51-61.

3. Savina S, Zaydiner B. Cancer-related fatigue: Some clinical aspects. Asia Pac J Oncol Nurs 2019; 6:7-9.

4. Hampson JP, Zick SM, Khabir T, et al. Altered resting brain connectivity in persistent cancer related fatigue. Neuroimage Clin 2015; 8:305-313.

5. O'Higgins CM, Brady B, O'Connor B, et al. The pathophysiology of cancer-related fatigue: Current controversies. Support Care Cancer 2018; 26:3353-3364.

6. Berger AM, Mooney K, Alvarez-Perez A, et al. Cancerrelated fatigue. J Natl Compr Canc Netw 2015; 13:10121039.

7. Vollestad NK. Measurement of human muscle fatigue. J Neurosci Methods 1997; 74:219-227.

8. Zwarts MJ, Bleijenberg G, van Engelen BGM. Clinical neurophysiology of fatigue. Clin Neurophysiol 2008; 119: $2-10$.

9. De Groef A, Van Kampen M, Tieto E, et al. Arm lymphoedema and upper limb impairments in sentinel nodenegative breast cancer patients: A one year follow-up study. Breast 2016; 29:102-108.

10. Chatterjee M, Murthy V, Gupta T, et al. Effect of progressive resistive exercises on shoulder abduction range of motion in post operative oral cancer patients undergoing radiation therapy. Physiotherapy 2015; 101:e216-e217.

11. Chen X, Stubblefield MD, Custodio CM, et al. Electrophysiological features of taxane-induced polyneuropathy in patients with breast cancer. J Clin Neurophysiol 2013; 30: 199-203.

12. Verstappen CC, Koeppen S, Heimans JJ. Dose-related vincristine-induced peripheral neuropathy with unexpected off-therapy worsening. Neurology 2005; 64:1076-1077.

13. de Witte PB, Werner S, ter Braak LM, et al. The Supraspinatus and the Deltoid-Not just two arm elevators. Hum Mov Sci 2014; 33:273-283.

14. Shamley D, Lascurain-Aguirrebeña I, Oskrochi R. Shoulder morbidity after treatment for breast cancer is bilateral and greater after mastectomy. Acta Oncol (Madr) 2012; 51: 1045-1053.

15. Shamley D, Srinaganathan R, Oskrochi R, et al. Threedimensional scapulothoracic motion following treatment for breast cancer. Breast Cancer Res Treat 2009; 118:315322.

16. Franc M, Michalski B, Kuczerawy I, et al. Cancer related fatigue syndrome in neoplastic diseases. Prz Menopauzalny $2014 ; 13: 352-355$.

17. Alves RS, Iunes DH, Pereira IC, et al. Influence of exergaming on the perception of cancer-related fatigue. Games Health J 2017; 6:119-126.

18. Cramp F, Byron-Daniel J. Exercise for the management of cancer-related fatigue in adults. Cochrane Database Syst Rev 2012; 16:1-97.

19. Huang X, Zhang Q, Kang X, et al. Factors associated with cancer-related fatigue in breast cancer patients undergoing endocrine therapy in an urban setting: A cross-sectional study. BMC Cancer 2010; 10:1-7.

20. Schmitz KH, Courneya KS, Matthews C, et al. American College of Sports Medicine roundtable on exercise 
guidelines for cancer survivors. Med Sci Sports Exerc 2010; 42:1409-1426.

21. Kuys SS, Hall K, Peasey M, et al. Gaming console exercise and cycle or treadmill exercise provide similar cardiovascular demand in adults with cystic fibrosis: A randomised cross-over trial. J Physiother 2011; 57:35-40.

22. Harvard Health Publishing. Fun and exergames: Not just for kids anymore. 2012. www.health.harvard.edu/hearthealth/fun-and-exergames-not-just-for-kids-anymore (accessed September 29, 2018).

23. Oliveira PF, Iunes DH, Alves RS, et al. Effects of exergaming in cancer related fatigue in the quality of life and electromyography of the middle deltoid of people with cancer in treatment: A controlled trial. Asian Pac J Cancer Prev 2018; 19:2591-2597.

24. Grange RW, Houston ME. Simultaneous potentiation and fatigue in quadriceps after a 60-second maximal voluntary isometric contraction. J Appl Physiol 1991; 70: 726-731.

25. Heinonen A, Sievänen H, Viitasalo J, et al. Reproducibility of computer measurement of maximal isometric strength and electromyography in sedentary middle-aged women. Eur J Appl Occup Physiol 1994; 68:310-314.

26. Martins J, Napoles BV, Hoffman CB, et al. The Brazilian version of Shoulder Pain and Disability Index: Translation, cultural adaptation and reliability. Braz J Phys Ther 2010; 14:527-536.

27. Satpute KH, Bhandari P, Hall T. Efficacy of hand behind back mobilization with movement for acute shoulder pain and movement impairment: A randomized controlled trial. J Manipulative Physiol Ther 2015; 38:324-334.

28. Mannion K, Stevens K, Murphy B, et al. Assessment of musculoskeletal impairment in head-and-neck cancer patients. Int J Radiat Oncol Biol Phys 2014; 88:529_ 530.

29. Fung YK, Choi WW, Lo TC, et al. Efficacy of modified 3 phases exercise program for patient following breast cancer surgery at Tseung Kwan O Hospital. Hong Kong Physiother J 2011; 29:98-99.

30. Jie H, Lingfeng $X$, Xiaoling $H$, Xiaohua H. Effects of Mulligan's mobilization with movement combined with stretching therapy in the management of frozen shoulder. Physiotherapy 2015; 101:e683-e684.

31. Clausen MB, Witten A, Holm KH, et al. Patient reported shoulder function in patients with subacromial impingement: The role of maximum isometric force, range of motion and pain. Physiotherapy 2015; 101:e257-e258.
32. Desmeules F, Boudreault J, Roy JS, et al. Efficacy of transcutaneous electrical nerve stimulation for rotator cuff tendinopathy: A systematic review. Physiotherapy 2016; 102:41-49.

33. Yan H, Pei G, Xiaoju Z. The effectiveness of rehabilitation program on shoulder function of breast cancer patients after modified radical mastectomy. JBI Libr Syst Rev 2005; 3: $1-17$.

34. Rietman JS, Dijkstra PU, Hoekstra HJ, et al. Late morbidity after treatment of breast cancer in relation to daily activities and quality of life: A systematic review. Eur J Surg Oncol 2003; 29:229-238.

35. Nijs J, Van Houdenhove B, Oostendorp RAB. Recognition of central sensitization in patients with musculoskeletal pain: Application of pain neurophysiology in manual therapy practice. Man Ther 2010; 15:135-141.

36. Louw A, Puentedura EJ, Reese D, et al. Immediate effects of mirror therapy in patients with shoulder pain and decreased range of motion. Arch Phys Med Rehabil 2017; 89:1941-1947.

37. Dahl-Popolizio S, Loman J, Cordes CC. Comparing outcomes of Kinect videogame-based occupational/physical therapy versus usual care. Games Health J 2014; 3:157-161.

38. Gemmell H, Miller P, Jones-Harris A, et al. An alternative approach to the diagnosis and management of non-specific shoulder pain with case examples. Clin Chiropr 2011; 14:38-45.

39. Gewandter JS, Chaudari J, Ibegbu C, et al. Wireless transcutaneous electrical nerve stimulation device for chemotherapy-induced peripheral neuropathy: An openlabel feasibility study. Support Care Cancer 2018; 1:1-10.

40. Prinsen H, van Dijk JP, Zwarts MJ, et al. The role of central and peripheral muscle fatigue in postcancer fatigue: A randomized controlled trial. J Pain Symptom Manage 2015; 49:173-182.

41. Wiskemann J, Schmidt ME, Klassen O, et al. Effects of 12week resistance training during radiotherapy in breast cancer patients. Scand J Med Sci Sports 2017; 27:1500-1510.

Address correspondence to: Ricardo da Silva Alves, MSc Bioscence Program Federal University of Alfenas 2600, Jovino Fernandes Sales Avenue Alfenas 37.133-840 Minas Gerais Brazil

E-mail: ricardofisio31@gmail.com 